\title{
Design, Construction and Test of Small-Diameter Muon Drift Tube Chambers (sMDT) for the Phase-1 Upgrade of the ATLAS Muon Spectrometer
}

\author{
Patrick Rieck, on behalf of the ATLAS collaboration \\ Max-Planck-Institute for Physics, Munich, Germany
}

\begin{abstract}
The ATLAS muon spectrometer consists of an efficient muon trigger system and high muon momentum resolution up to the $\mathrm{TeV}$ scale. Yet, in the regions between the inner barrel and endcap of the muon spectrometer the trigger selectivity is limited. Furthermore, at the future High-Luminosity LHC the efficiency of the resistive plate trigger chambers (RPCs) will decrease due to ageing effects. Therefore, additional RPCs will be installed at the ends of the inner barrel layer of the muon spectrometer in the current long shutdown for the Phase-1 upgrade of the LHC in 2019 and 2020. In order to free space for them, the current Muon Drift Tube (MDT) chambers will be replaced by small-diameter Muon Drift Tube (sMDT) chambers with 15 instead of 30 mm tube diameter, which will be integrated with thin-gap RPCs. Due to their higher background rate capability, the new sMDT chambers are also suitable precision muon tracking detectors at future hadron colliders. An overview of the design and production of the new ATLAS sMDT chambers, their performance and their mechanical integration with the RPCs is given. The construction of these new chambers also serves as a pilot project for the replacement of half of the barrel inner layer in the Phase-2 upgrade of the ATLAS detector.
\end{abstract}

Keywords: Muon chambers, ATLAS detector, small-diameter MDT chambers, thin-gap RPC chambers

\section{Upgrade Requirements}

In the ATLAS Muon Spectrometer, the acceptance provided by the RPCs used for triggering in the barrel part is limited by gaps due to inner detector and calorimeter services as well as toriod magnet structures. Furthermore, fake trigger rates are significant at both ends of the barrel part due to the high particle fluxes in these regions. Moreover, the large background radiation rates to which the detectors are exposed at the HighLuminosity LHC will require to operate the current RPCs at a reduced high voltage, which reduced the trigger efficiency [1].

In order to overcome these limitations, the current RPCs will be supplemented by triplets of new thin-gap RPCs in the innermost layer of the barrel muon spectrometer. The MDT chambers in the small sectors of the inner barrel layer (BIS) at the boundaries of the toroid magnet coils must be replaced for this purpose by new sMDT chambers integrated mechanically with the new RPCs. Apart from their compact design, the sMDT chambers also provide a rate capability which is increased by a factor of eight compared to the MDT chambers. As a pilot project, in the current long shutdown of the LHC the first 16 integrated RPC and sMDT BIS chambers will be installed [2].

\footnotetext{
${ }^{*}$ Corresponding author

Email address: prieck@mpp.mpg.de (Patrick Rieck, on behalf of the ATLAS collaboration)
}

\section{Drift Tube Design and Construction}

The drift tubes of the sMDT chambers have a diameter of $15 \mathrm{~mm}$, which is a factor of 2 smaller compared to the standard MDT chambers. The applied voltage of $2730 \mathrm{~V}$ between the anode wires and the tube walls corresponds to a gas gain of 20000. Like the MDT chambers, they are operated with a $\mathrm{Ar}: \mathrm{CO}_{2}$ (93:7) gas at a pressure of 3 bar. Apart from the more compact design, the diameter reduction also leads to a decrease of the background occupancy by a factor of eight due to the four times shorter maximum drift time and the two times smaller tube cross-section exposed to the photon and neutron background radiation in the ATLAS cavern. The reduction in drift time also allows for a shortening of the electronics dead time without an increase of hit rate and output bandwidth.

The $0.4 \mathrm{~mm}$ thick aluminium tubes are chromatised in order to ensure reliable electrical ground contacts. The tungstenrhenium sense wires with a diameter of $50 \mu \mathrm{m}$ are fixed in the insulating plastic endplugs at both ends of the tubes. The assembly of the tubes is performed by means of a semi-automated wiring station in a climatised clean room. The wires are inserted into the tubes and endplugs using clean air flow, fixed within crimped copper tubelets in the centres of the endplugs and tensioned to $350 \pm 15 \mathrm{~g}$. The wires are positioned with a precision of a few $\mu \mathrm{m}$ with respect to cylindrical external reference surface on the central brass insert of the endplugs (Fig. 11).

Subsequently, the quality of each tube is tested by measurements of the wire tension, the dark current under high voltage and the gas leakage rate. The latter is required to be less than $0.23 \mathrm{mbar} / \mathrm{h}$ for tubes of $1 \mathrm{~m}$ length. An overall failure rate of 


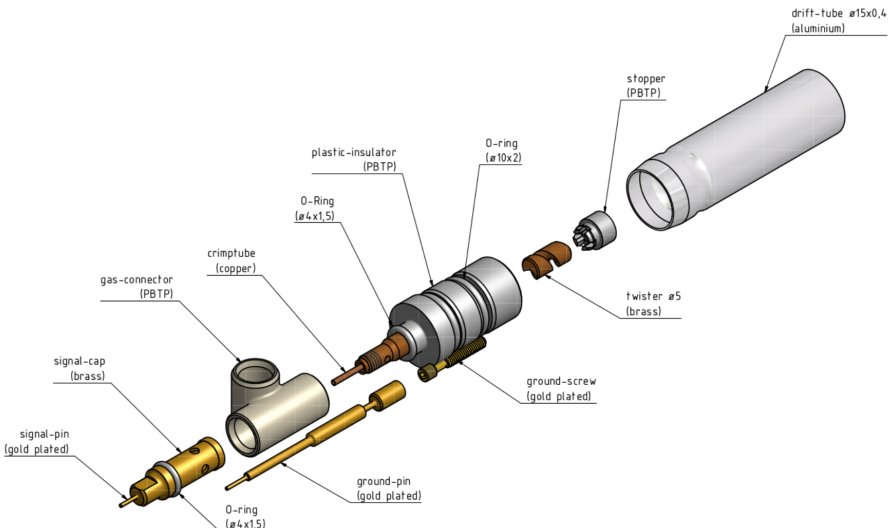

Figure 1: Composition of sMDT drift tube endplugs. The spiral wire locator (twister) precisely positions the sense wire with respect to the external reference surface on the brass insert of the injection-molded endplug. The injectionmolded gas connector and the gold-plated electrical connections, namely the signal pin and the ground pin connection to the tube walls, are also shown.

only $2 \%$ has been achieved. Failures are mostly due to dark currents greater than $2 \mathrm{nA} / \mathrm{m}$, which can be caused by imperfections of the tubes inner surface. An average tube production rate of 60 tubes per day is achieved with three technicians operating one assembly station, one wire tensioning station and one station for high voltage and gas leakage tests.

\section{Chamber Design and Construction}

Each chamber consists of two multilayers of drift tubes, each containing four tube layers. In order to maximise the coverage, 12 different variants of sMDT chambers are constructed. They are made of either 648 or 744 drift tubes and cover an area of up to $1.6 \mathrm{~m}^{2}$.

Drift tubes are assembled in a climatised clean room on a flat granite table [3]. Aluminium jigs with a precise grid of bores define the positions of the tube endplugs, and consequently of the sense wires, at each chamber end. The accuracy of the bore positions measured with a coordinate measuring machine is 5 $\mu \mathrm{m}$ and hence well below the required sense wire positioning accuracy in a chamber, which is $20 \mu \mathrm{m}$ (rms). The reference surfaces of the endplugs are placed within the bores, which results in a high positioning accuracy of the sense wires attached to the endplugs (Fig. 2). The tubes are glued together using an automated glue dispenser which deposits glue in the grooves between the tubes of each layer. Between successive tube layers, which are offset by half a tube diameter in the chamber plane with respect to each other, screws are inserted which connect each tube wall to the electronics ground via gold-plated ground pins. On top of the first multilayer, aluminium profiles are glued. On top of the second multilayer, support structures, platforms for magnetic field sensors and chamber alignment components are glued. The latter include platforms which serve as the base for optical alignment instruments which determine the position of the chamber within the ATLAS muon spectrometer. These platforms are mounted by means of assembly jigs which are positioned in relation to the aluminium

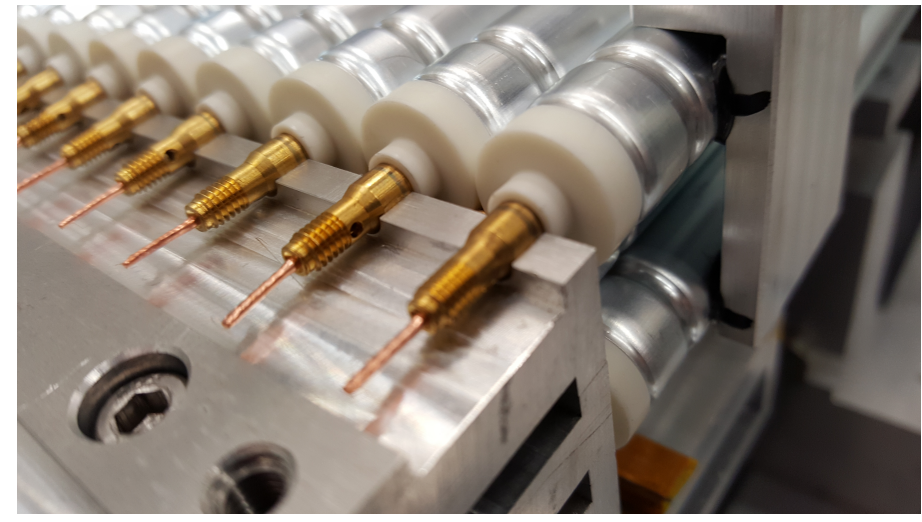

(a)

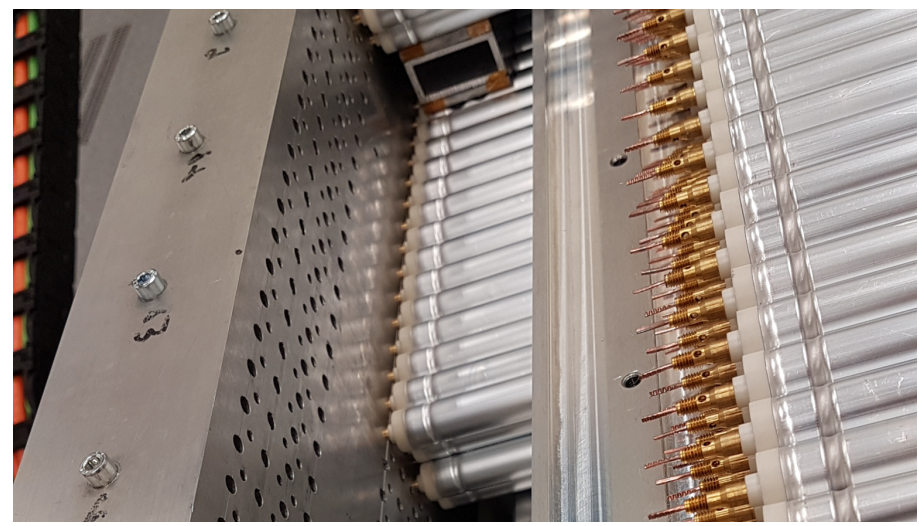

(b)

Figure 2: Drift tubes positioned by means of a precise aluminium comb. The reference surfaces on the drift tube endplugs fit into bores drilled into the aluminium comb. As the sense wires are positioned precisely with respect to the tube endplug reference surfaces, this assembly technique allows for a high mechanical accuracy of the detector. The aluminium combs also provide smaller bores which define the positions of electrical grounding screws connected to the tube walls.

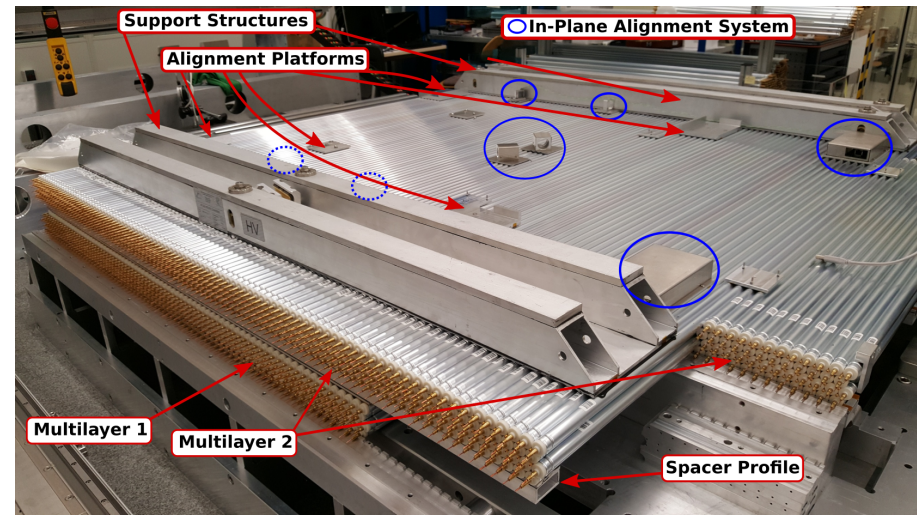

Figure 3: An assembled sMDT chamber. The two multilayers of drift tubes are separated by aluminium spacer profiles. Support structures are glued on top of the chamber at both of its ends. Platforms are provided to mount optical alignment instruments which determine the chamber position within the ATLAS muon spectrometer. An in-plane alignment system consisting of two CCD cameras covered in boxes on the right hand side, four lenses in the middle and two LEDs on the left hand side of the picture is used to monitor the deformation of the chamber during its operation. 

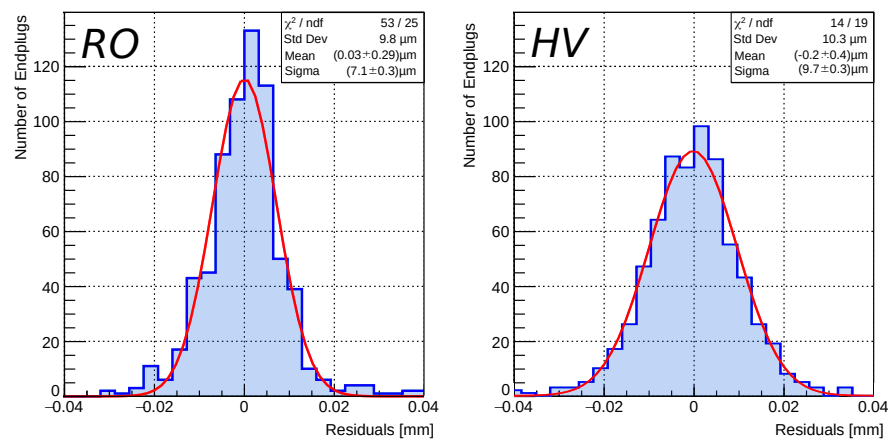

Figure 4: Residual distribution of the sense wire positions on the Readout (RO) and High-Voltage (HV) sides of the first BIS sMDT chamber, comparing measurements obtained using a CMM and ideal wire grid positions. The latter are derived from a fit to the CMM data including parameters on account of gravitational deformations. The accuracy of the sense wire positioning is below $10 \mu \mathrm{m}$, compared to the required accuracy of $20 \mu \mathrm{m}$.

combs which define the sense wire positions. Furthermore, an in-plane alignment system consisting of LEDs, lenses and CCD cameras which monitor four optical paths across the chamber surface in order to determine the gravitational deformation of the chamber is glued on top of the second multilayer as well. An assembled chamber on the granite table with the aluminium jigs removed is shown in Fig. 3

\section{Mechanical Accuracy}

The wire positions at both ends of a prototype BIS sMDT chambers has been measured with a large coordinate measuring machine (CMM). The measurement makes use of the cylindrical reference surfaces of the drift tube endplugs. An ideal wire grid with the wire pitches and the relative multilayer displacements in the two directions perpendicular to the tubes as free parameters is fitted to the measured wire positions. Also gravitational deformations at both ends of the chamber are taken into account. The resulting wire positions agree well with the nominal values. The overall accuracy of the sense wire positioning is below $10 \mu \mathrm{m}$ (Fig 4 ).

During the serial chamber production, the sense wire positions in the coordinate perpendicular to the chamber plane are evaluated by measuring the endplug reference surface positions with a feeler gauge above a flat granite table with a precision of a few $\mu \mathrm{m}$. This procedure also allows to validate the measurement of gravitational sags and torsions provided by the inplane alignment system, which is operated during the feeler gauge measurements. The agreement between mechanical and in-plane alignment measurements is on the level of $50 \%$, which is sufficient in order not to deteriorate the muon track point measurement accuracy.

Also for every chamber the positions of the alignment platforms are determined by means of a measurement arm, which results in a positioning accuracy on the level of $100 \mu \mathrm{m}$ in the coordinates perpendicular to the tube axes.

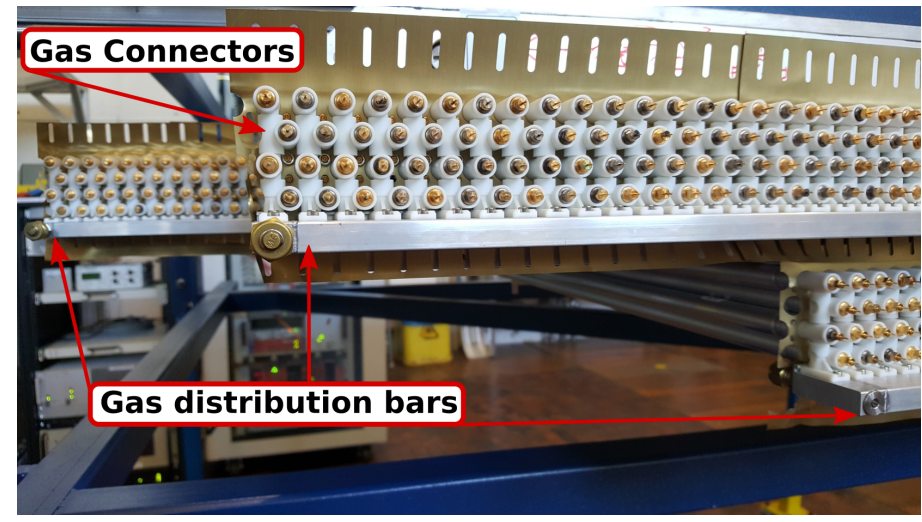

Figure 5: Gas distribution system with injection molded plastic gas connectors mounted on each endplug. They interconnect the drift tubes and link them to the gas distribution bars.

\section{Gas Distribution System}

The sMDT chambers are operated with an $\mathrm{Ar}: \mathrm{CO}_{2}$ (93:7) gas mixture at a pressure of 3 bar as the ATLAS MDT chambers. The gas leakage rate of the chambers is tightly constrained to less than $2 \cdot 10^{-8}$ bar $1 / \mathrm{s}$, which corresponds to a pressure drop of $0.4 \mathrm{mbar} / \mathrm{h}$ for a chamber gas volume of 1701 , in order to avoid any contamination of the drift gas.

The gas supply to each drift tube is realised by injection molded plastic gas connectors on the endplugs (Fig. 1), which are interconnected in the direction perpendicular to the tubes of each multilayer and link the drift tubes to gas distribution bars mounted on both ends of the chamber in each multilayer (Fig. 5).

\section{Electronics Commissioning}

High-voltage and signal distribution boards are mounted on opposing ends of the chambers. Each board serves $6 \times 4$ drift tubes in a multilayer. Decoupling capacitors and termination resistors are separated by plastic insulators in order to prevent discharges in the dense grid of endplugs under high voltage. The signal distribution boards carry stacked active mezzanine cards which in their first layer contain three 8-channel AmplifierShaper-Discriminator (ASD) chips of the type already used in the ATLAS MDT chambers [4]. The discriminator output signals are processed by a high performance TDC (HPTDC) with a time resolution of $0.2 \mathrm{~ns}$, which was developed at CERN [5]. The TDC board also contains an FPGA which is used for setting the programmable chip parameters via the JTAG protocol. The TDC timing data of the signal arrival time are sent to a chamber service module (CSM) where they are multiplexed and transmitted to the ATLAS data acquisition system.

Chromatised $0.2 \mathrm{~mm}$ thick aluminium foils and the grounding screws electrically interconnect all tube walls in a multilayer. Finally, Faraday cages enclose the high-voltage and readout electronics. The active mezzanine cards are separated from the signal distribution board by a shielding plane which is part of the Faraday cage. A schematic view and a photograph of the readout electronics are shown in Fig. 6a and 6b, respectively. 


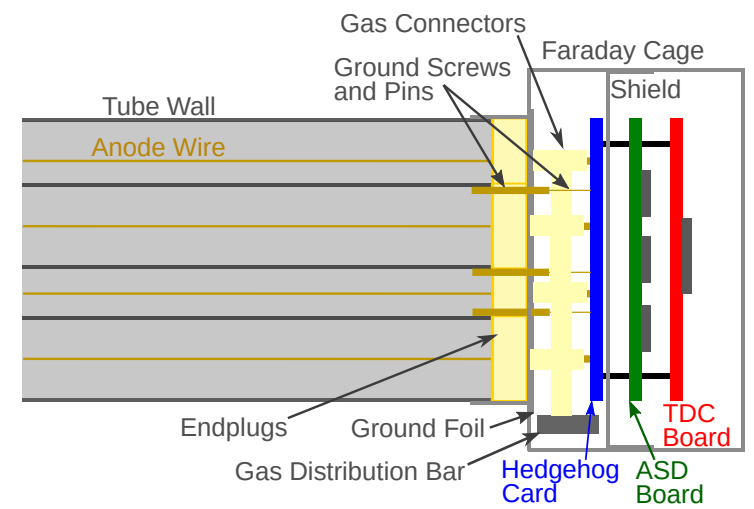

(a)

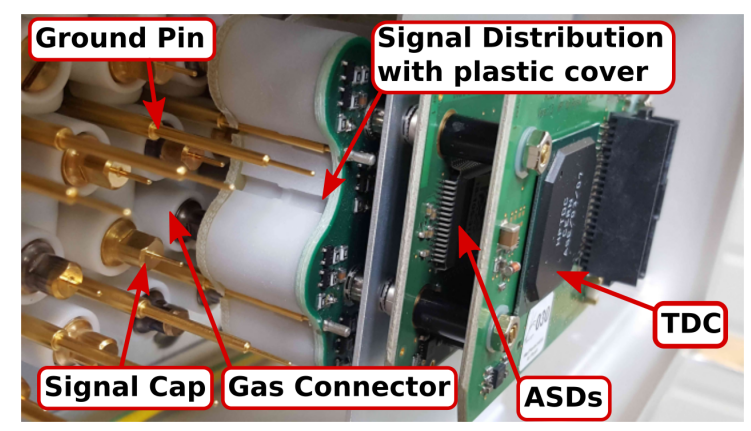

(b)

Figure 6: Schematic view of the readout electronics $6 \mathrm{a}$ and photograph of a signal board on an sMDT chamber 6b. In the latter, the plastic cover encapsulating coupling capacitors and termination resistors is used to prevent discharges under high voltage.

The electronics performance on the chamber is tested with respect to dark currents at operating voltage, accidental counting rates, shape of the TDC spectra, muon detection efficiency and drift tube spatial resolution with cosmic muon tracks.

Before the mezzanine cards are mounted on a chamber, the noise rate of each channel is tested using a dedicated setup. Inside a Faraday cage, each signal board is connected directly to a high voltage distribution board and the noise rate is measured, providing the references of minimum noise rate to be expected depending on the individual discriminator threshold on the ASD chips. Comparing these noise rates with noise measurements on the chambers, negligible difference was found.

\section{RPC Integration}

Given the smallness of the space available for the BIS sMDT and RPC chambers, an integrated mechanical design of the two chamber types with low tolerances is required (Fig. 7). The separated supports of the sMDT and RPC chambers are adjusted under the installation angle depending on the assigned sector in the barrel muon spectrometer just before installation using a rotation frame.

\section{Conclusions}

New integrated sMDT precision muon tracking and RPC muon trigger chambers are constructed for the Phase-1 upgrade

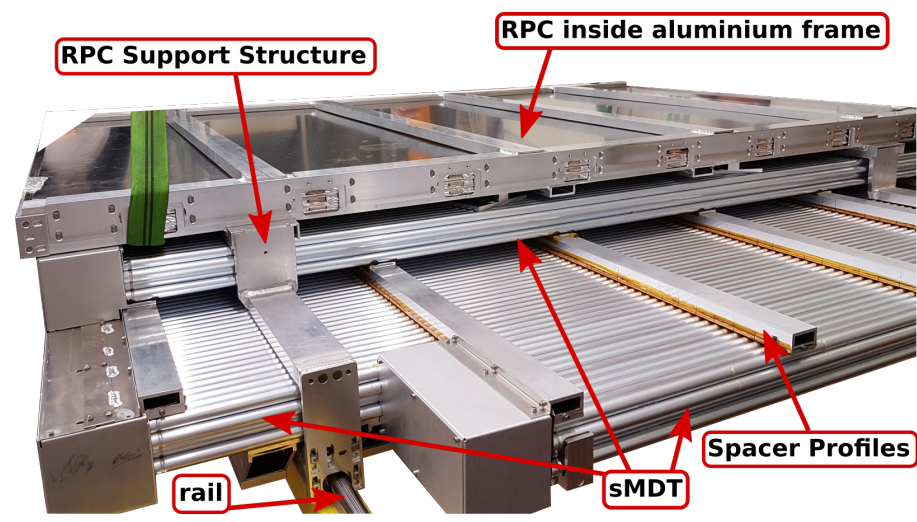

Figure 7: Mechanical integration of a BIS sMDT and RPC chamber. The three RPC layers are enclosed in an aluminium frame which holds them together and limits gravitational sag. The sMDT and RPC chambers are mounted separately on the rails in the ATLAS detector via interleaved support structures.

of the ATLAS barrel muon spectrometer, in order to increase the trigger acceptance and to prepare for the high background rate requirements of the High-Luminosity LHC. The SMDT chambers are built with a high mechanical accuracy, gas distribution systems with low leakage rates and low noise electronics. The transport of sMDT chambers to CERN has started, where they will be integrated with RPC chambers for installation in the ATLAS muon spectrometer starting in summer 2019.

Copyright 2019 CERN for the benefit of the ATLAS Collaboration. CC-BY-4.0 license.

\section{References}

[1] The ATLAS Collaboration, Technical Design Report for the Phase-II Upgrade of the ATLAS Muon Spectrometer (CERN-LHCC-2017-017. ATLAS-TDR-026). URL https://cds.cern.ch/record/2285580

[2] H. Kroha, Design and construction of integrated small-diameter drift tube and thin-gap resistive plate chambers for the phase-1 upgrade of the ATLAS muon spectrometer, in: 14th Pisa Meeting on Advanced Detectors: Frontier Detectors for Frontier Physics (Pisameet) La Biodola-Isola d'Elba, Livorno, Italy, May 27-June 2, 2018.

[3] H. Kroha, R. Fakhrutdinov, O. Kortner, A. Kozhin, K. SchmidtSommerfeld, E. Takasugi, New High-Precision Drift-Tube Detectors for the ATLAS Muon Spectrometer, JINST 12 (06) (2017) C06007. arXiv: 1705.05656 doi:10.1088/1748-0221/12/06/C06007

[4] C. Posch, E. Hazen, J. Oliver, MDT-ASD, CMOS front-end for ATLAS MDT; rev. version 2.1. Tech. Rep. ATL-MUON-2002-003, CERN, Geneva, revised version number 2.1 was submitted on 2007-09-18 19:14:00 (Sep 2007). URL http://cds.cern.ch/record/684217

[5] J. Christiansen, HPTDC High Performance Time to Digital Converter Tech. rep., CERN, Geneva, version 2.2 for HPTDC version 1.3 (2004). URL https://cds.cern. ch/record/1067476 